\title{
Insights into the inflammatory process of lumbar discopathy
}

\author{
Andreas K. Demetriades ${ }^{1}$ (D) \\ Received: 21 October 2019 / Accepted: 23 October 2019 / Published online: 12 November 2019 \\ (C) Springer-Verlag GmbH Austria, part of Springer Nature 2019
}

It is postulated that a prolapsed lumbar disc may produce compressive symptoms as well as an inflammatory frictional effect. The role of macrophage tissue infiltration in this process has long been suspected, but difficult to pin down [3].

Twenty years later, a recent systematic review has found it difficult to "draw firm conclusions regarding the relationship between inflammation and clinical symptoms with sciatica" [2].

Animal studies have indeed shown that a lumbar disc herniation can activate the expression of IGF-1 initially, followed by activation of the PI3K/AKT signaling pathway, leading to the expression of IL-1 and IL-6 inflammation-related factors. This may represent the body's attempt at tissue repair and the regulation of cytokines and the apoptotic process [4].

Colleagues from the Netherlands have investigated the role of macrophages in sciatica, using a retrospective observational histological study [1]. They looked for associations between the size and type of lumbar disc herniation on MRI, Modic changes in the vertebral endplates, and the degree of macrophage infiltration in a group of 119 patients. They clearly describe the knowledge gap in their introduction, including the questioning of what role might macrophages play; what might Modic changes suggest for the inflammatory process in the disc space; whether Modic changes suggest a slower recovery from disc herniation; and whether this is related to the resorption process.

Their results show that:

- at baseline, the degree of macrophage infiltration was not associated with the size of disc herniation.

This article is part of the Topical Collection on Spine degenerative

Andreas K. Demetriades

andreas.demetriades@gmail.com

1 Department of Neurosurgery, Western General Hospital,

Edinburgh, UK
- however, the degree of macrophage infiltration was significantly associated with a reduction of the size of disc herniation at one year postop.

- the degree of macrophage infiltration was higher in extrusion rather than in disc protrusion.

This interestingly suggests that patients with extruded disc fragments might benefit from a prolonged conservative therapy, whereby the increased macrophage response can be at play. The authors suggest that this might be because extruded disc fragments are more exposed to the systemic circulation; are they really, given that they are disconnected from the endplate? Could the reverse be true, that if cut off from the systemic circulation, an extruded fragment might atrophy faster than a prolapsed fragment?

Another interesting finding was that results were comparable in patients with and without Modic changes, the study size sample being too small to go beyond any suggestion of a trend of significant association.

There are important limitations to this study, described well by the authors themselves, not least that macrophages are only but a part of the inflammatory cascade and its relation to neovascularisation. Their conclusion, that macrophage infiltration was positively associated with an extruded type of disc herniation as well as the extent of reduction of the herniated disc during 1-year follow-up, opens possibilities for further work in the area. 


\section{References}

1. Djuric N, Yang X, elBarzouhi et al (2019) Lumbar disc extrusions reduce faster than bulging disc due to an active role of macrophages in sciatica. Acta Neurochir (in press)

2. Jungen MJ, Ter Meulen BC, van Osch T, Weinstein HC, Ostelo RWJG (2019) Inflammatory biomarkers in patients with sciatica: a systematic review. BMC Musculoskelet Disord 20:156. https://doi. org/10.1186/s12891-019-2541-0

3. Rothoerl R, Woertgen C, Holzschuh M, Brehme K, Rüschoff J, Brawanski A (1998) Macrophage tissue infiltration, clinical symptoms, and signs in patients with lumbar disc herniation. A clinicopathological study on 179 patients. Acta Neurochir 140(12): $1245-1248$

4. Xu Z, Zhou X, Chen G (2019) Expression and mechanism of interleukin 1 (IL-1), interleukin 2 (IL-2), interleukin 8 (IL-8), BMP, fibroblast growth factor 1 (FGF1), and insulin-like growth factor (IGF1) in lumbar disc herniation. Med Sci Monit 25:984-990

Publisher's note Springer Nature remains neutral with regard to jurisdictional claims in published maps and institutional affiliations. 